\title{
A Free-Flight Simulation of Insect Flapping Flight
}

\author{
Masateru MAEDA ${ }^{\mathrm{a}}, \mathrm{Na} \mathrm{GAO}^{\mathrm{a}}$, Naoshi NISHIHASHI ${ }^{\mathrm{a}}$, and Hao LIU ${ }^{\mathrm{a},{ }^{*}}$ \\ ${ }^{a}$ Graduate School of Engineering, Chiba University, Japan.
}

\begin{abstract}
In insect flapping flight, non-equilibrium flight conditions such as takeoffs and uncoordinated turn are difficult to investigate with experiments or quasi-steady analysis. Here we develop a simplified rigid body dynamics solver with 6 degrees of freedom (DOF), by utilizing unit quaternions. A free-flight simulator of an insect flapping flight is then built up by coupling the dynamics solver with an in-house CFD solver, which is specified for simulating unsteady flapping-wing aerodynamics. Simulation of the hovering flight of a fruit fly (Drosophila melanogaster) is achieved by manually manipulating three kinematic parameters, wingbeat amplitude, mean positional angle, and stroke plane angle relative to body (anatomical stroke plane angle).
\end{abstract}

Index Terms - flapping-flight, insect flight, free-flight, computational fluid dynamics, rigid body dynamics

\section{INTRODUCTION}

In numerical simulations of the flapping flight of animals or MAVs (micro air vehicles), improved quasisteady aerodynamic modeling is sometimes employed [1-3]. However, solving full Navier-Stokes equations is necessary to obtain higher fidelity flow field information around an insect, from which we can directly compute the aerodynamic forces and torques (moments) acting on the insect. Liu and colleagues [46] carry out several numerical simulations on insects flapping flight using such a computational fluid dynamic (CFD) solver. All of the researches, however, treats tethered-flight conditions; that is, the body of the insect is fixed in space. Ramamurti and Sandberg [7] numerically investigate a maneuver called saccade, a sharp turn, of fruit flies. The unsteady aerodynamic forces and torques are computed, but the wing and body kinematics are prescribed from observed behaviour. So it is not possible to know, for example, "after that" flight sequences or "what if" situations.

Sun and colleagues [8] circumvent this problem by coupling fluid dynamics and rigid body dynamics. However, since their solver has only three degrees of freedom (DOF), their work is limited to either longitudinal [9] or lateral motion [10].

Therefore, if one wants to numerically investigate non-equilibrium flight conditions such as take-off [11-

\footnotetext{
* Corresponding author: 1-33 Yayoi-cho, Inage-ku, Chiba 263-8522, Japan. E-mail: hliu@faculty.chiba-u.jp
}

12] or flight in a gusty environment [13-14], an unsteady, 6DOF free-flight simulator is needed. We have developed a simplified rigid body dynamics (RBD) solver in terms of 6DOF, in which we utilised unit quaternion for attitude description. By combining the RBD solver with an in-house CFD solver [17], we have established a 6DOF free-flight simulator, which is capable to simulate transient flight dynamics and aerodynamics in insect flapping flight. The overall formulation is similar to that of Kawamura et al. [15].

In this paper, we briefly explain the derivation of the simplified RBD solver and coupling method. As a preliminary computation, the free-flight of a fruit fly hovering is realized by adjusting three kinematic parameters manually.

\section{METHODS}

The simulator consists of two major components, a fluid dynamic solver and a simplified rigid body dynamic solver.

\section{A. Flow solver}

Flow fields are computed using the unsteady 3D incompressible Navier-Stokes solver developed by Liu et al. [16-17], which utilizes pseudo-compressibility and gird oversetting method. The details of the flow solver are provided elsewhere [17].

There are two dimensionless numbers used in the computation, namely, the Reynolds number Re and the reduced frequency $k$, which characterize the flow field. The Reynolds number is defined as

$$
\mathrm{Re}=\frac{c_{\mathrm{m}} u_{\mathrm{ref}}}{v},
$$

where $c_{\mathrm{m}}$ is mean chord length, $v$ is the kinematic viscosity of air, and $u_{\text {ref }}$ is reference velocity. In hovering flight, reference velocity is calculated as

$$
u_{\text {ref }}=2 \Phi R f \text {, }
$$

where $\Phi$ is wing beat amplitude, $R$ is the length of a wing (from root to tip), and $f$ is wingbeat frequency. Reduced frequency is defined as

$$
k=\frac{\omega c_{\mathrm{m}}}{2 u_{\mathrm{ref}}}=\frac{2 \pi f c_{\mathrm{m}}}{2 u_{\mathrm{ref}}}=\frac{\pi f c_{\mathrm{m}}}{u_{\mathrm{ref}}},
$$

where $\omega=2 \pi f c_{\mathrm{m}}$ is angular frequency. 


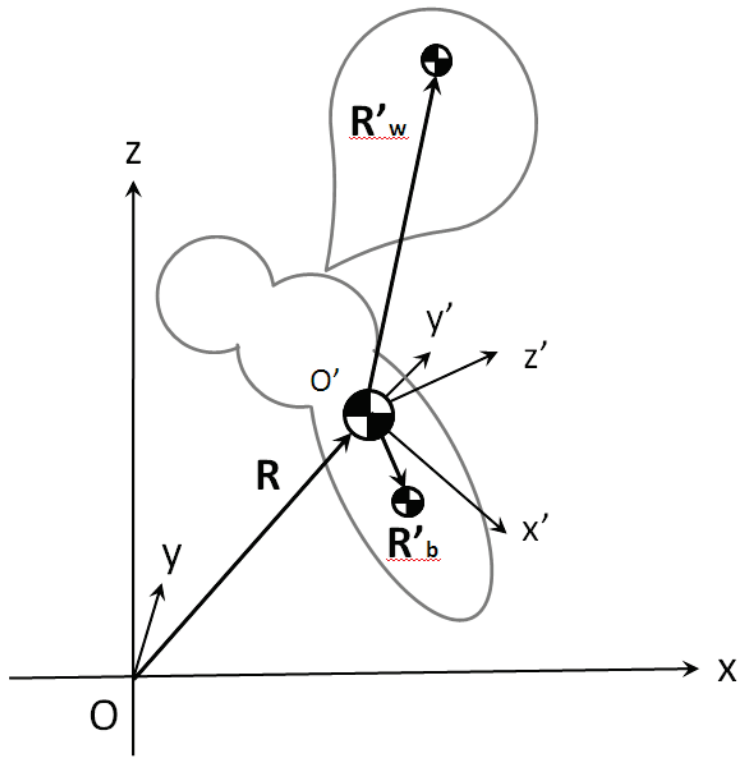

Fig. 1 Schematics of frames and vectors. (x, y, z), inertial frame of reference, "global coordinates" in short. (x', y', z'), principal axes of inertia, "body coordinates" in short. $\mathbf{R}$ is the position vector of the flier's center of mass in global coordinates. $\mathbf{R}_{\mathbf{b}}{ }_{\mathbf{b}}$ and $\mathbf{R}_{{ }_{\mathbf{w}}}$ are the vectors of the body and wings, respectively, in body coordinates.

\section{B. Simplified rigid body dynamics solver}

A simplified rigid body dynamics (RBD) solver was developed to obtain insect body motion.

Several simplifications were introduced which make the equations of motion simpler and the implementation of the programming code easier. Translational equations of motion are described in the inertial frame of reference and rotational equations of motion are described with respect to the principal axes of inertia of the flier (Fig. 1). Coordinates in the inertial frame of reference are hereafter called "global coordinates." The principal axes of inertia are approximated by the body axes and are hereafter called "body coordinates." Since an insect flaps its wings, a time-averaged value is used for the moment of inertia, which is given in body coordinates, around the center of mass (CM) of the flier. Unlike the work of Sun et al. [8], both the translational and rotational movements are described around total center of mass, not the body center of mass. Therefore, the body oscillation due to flapping does not appear in the equations of motion. Instead, the oscillating motion is implemented separately. This is explained later.

The equations of motion can be written as [18]:

$$
\begin{aligned}
& M £=\mathbf{F}_{\mathrm{a}}+\mathbf{F}_{\mathrm{g}}, \\
& \mathbf{J}^{\prime} \boldsymbol{\Phi}+\tilde{\mathbf{\Omega}}^{\prime} \mathbf{J}^{\prime} \mathbf{\Omega}^{\prime}=\mathbf{N}_{\mathrm{a}}^{\prime},
\end{aligned}
$$

where $M$ is the total mass of the flier, $\mathbf{V}=\left[V_{\mathrm{x}}, V_{\mathrm{y}}, V_{\mathrm{z}}\right]^{T}$ is translational velocity at flier's CM, $\quad \mathbf{F}_{\mathrm{a}}=\left[F_{\mathrm{ax}}, F_{\mathrm{ay}}, F_{\mathrm{az}}\right]^{T}$ is aerodynamic force acting on the flier, $\mathbf{F}_{\mathrm{g}}=[0,0,-M g]^{T}$ is gravitational force, where $g$ is gravitational acceleration at sea level,

$$
\mathbf{J}^{\prime}=\left[\begin{array}{ccc}
J_{\mathrm{xx}}^{\prime} & J_{\mathrm{xy}}^{\prime} & J_{\mathrm{xz}}^{\prime} \\
J_{\mathrm{yx}}^{\prime} & J_{\mathrm{yy}}^{\prime} & J_{\mathrm{yz}}^{\prime} \\
J_{\mathrm{zx}}^{\prime} & J_{\mathrm{zy}}^{\prime} & J_{\mathrm{zz}}^{\prime}
\end{array}\right] \approx\left[\begin{array}{ccc}
J_{\mathrm{x}}^{\prime} & 0 & 0 \\
0 & J_{\mathrm{y}}^{\prime} & 0 \\
0 & 0 & J_{\mathrm{z}}^{\prime}
\end{array}\right],
$$

is cycle-averaged moment of inertia tensor, $\boldsymbol{\Omega}^{\prime}=\left[\Omega_{\mathrm{x}}^{\prime}, \Omega_{\mathrm{y}}^{\prime}, \Omega_{\mathrm{z}}^{\prime}\right]^{T} \quad$ is angular velocity, and $\mathbf{N}_{\mathrm{a}}^{\prime}=\left[N_{\mathrm{ax}}^{\prime}, N_{\mathrm{ay}}^{\prime}, N_{\mathrm{az}}^{\prime}\right]^{T}$ is aerodynamic torque. Note that the variables with primes are in body coordinates, whereas those without primes are in global coordinates. The terms with dots are time derivatives. The tilde over the angular velocity term is an operator to create a $3 \times 3$ skew symmetric matrix from a $3 \times 1$ matrix:

$$
\tilde{\boldsymbol{\Omega}}^{\prime}=\left[\begin{array}{ccc}
0 & -\Omega_{\mathrm{z}}^{\prime} & \Omega_{\mathrm{y}}^{\prime} \\
\Omega_{\mathrm{z}}^{\prime} & 0 & -\Omega_{\mathrm{x}}^{\prime} \\
-\Omega_{\mathrm{y}}^{\prime} & \Omega_{\mathrm{x}}^{\prime} & 0
\end{array}\right] .
$$

Accompanying equations are needed to complete the rigid body dynamics. For translational motion, the equation for position is

$$
\hat{F}=\mathbf{V} \text {, }
$$

where $\mathbf{R}=\left[R_{\mathrm{x}}, R_{\mathrm{y}}, R_{\mathrm{z}}\right]^{T}$ is the flier center of mass in global coordinates. For attitude description, a set of unit quaternions (also known as Euler parameters) $\varepsilon_{0}{ }^{2}, \varepsilon_{1}{ }^{2}, \varepsilon_{2}{ }^{2}, \varepsilon_{3}{ }^{2}$ is introduced so that singular attitude ("gimbal lock") is avoided. To maintain three rotational degrees of freedom, an additional constraint equation (9) has to be satisfied:

$$
\varepsilon_{0}^{2}+\varepsilon_{1}^{2}+\varepsilon_{2}^{2}+\varepsilon_{3}^{2}=1 .
$$

The equations for attitude are

$$
\mathbf{E}=\frac{1}{2} \mathbf{S}^{T} \mathbf{\Omega}^{\prime},
$$

where $\mathbf{E}=\left[\varepsilon_{0}, \varepsilon_{1}, \varepsilon_{2}, \varepsilon_{3}\right]^{T}$ is a set of unit quaternion and $\mathbf{S}$ is the matrix,

$$
\mathbf{S}=\left[\begin{array}{rrrr}
-\varepsilon_{1} & \varepsilon_{0} & \varepsilon_{3} & -\varepsilon_{2} \\
-\varepsilon_{2} & -\varepsilon_{3} & \varepsilon_{0} & \varepsilon_{1} \\
-\varepsilon_{3} & \varepsilon_{2} & -\varepsilon_{1} & \varepsilon_{0}
\end{array}\right] .
$$

Eventually, the following system of equations is obtained: 


$$
\begin{aligned}
& \frac{\mathrm{d}}{\mathrm{d} t_{\mathrm{dyn}}}\left[\begin{array}{c}
R_{\mathrm{x}} \\
R_{\mathrm{y}} \\
R_{\mathrm{z}}
\end{array}\right]=\left[\begin{array}{c}
V_{\mathrm{x}} \\
V_{\mathrm{y}} \\
V_{\mathrm{z}}
\end{array}\right], \\
& \frac{\mathrm{d}}{\mathrm{d} t_{\mathrm{dyn}}}\left[\begin{array}{c}
V_{\mathrm{x}} \\
V_{\mathrm{y}} \\
V_{\mathrm{z}}
\end{array}\right]=\left[\begin{array}{c}
F_{\mathrm{ax}} / M \\
F_{\mathrm{ay}} / M \\
F_{\mathrm{az}} / M-g
\end{array}\right], \\
& \frac{\mathrm{d}}{\mathrm{d} t_{\mathrm{dyn}}}\left[\begin{array}{c}
\varepsilon_{0} \\
\varepsilon_{1} \\
\varepsilon_{2} \\
\varepsilon_{3}
\end{array}\right]=\frac{1}{2}\left[\begin{array}{c}
-\varepsilon_{1} \Omega_{\mathrm{x}}^{\prime}-\varepsilon_{2} \Omega_{\mathrm{y}}^{\prime}-\varepsilon_{3} \Omega_{\mathrm{z}}^{\prime} \\
\varepsilon_{0} \Omega_{\mathrm{x}}^{\prime}-\varepsilon_{3} \Omega_{\mathrm{y}}^{\prime}+\varepsilon_{2} \Omega_{\mathrm{z}}^{\prime} \\
\varepsilon_{3} \Omega_{\mathrm{x}}^{\prime}+\varepsilon_{0} \Omega_{\mathrm{y}}^{\prime}-\varepsilon_{1} \Omega_{\mathrm{z}}^{\prime} \\
-\varepsilon_{2} \Omega_{\mathrm{x}}^{\prime}+\varepsilon_{1} \Omega_{\mathrm{y}}^{\prime}+\varepsilon_{0} \Omega_{\mathrm{z}}^{\prime}
\end{array}\right], \\
& \frac{\mathrm{d}}{\mathrm{d} t_{\mathrm{dyn}}}\left[\begin{array}{c}
\Omega_{\mathrm{x}}^{\prime} \\
\Omega_{\mathrm{y}}^{\prime} \\
\Omega_{\mathrm{z}}^{\prime}
\end{array}\right]=\left[\begin{array}{l}
{\left[\left(J_{\mathrm{y}}^{\prime}-J_{\mathrm{z}}^{\prime}\right) \Omega_{\mathrm{y}}^{\prime} \Omega_{\mathrm{z}}^{\prime}+N_{\mathrm{ax}}^{\prime}\right] / J_{\mathrm{x}}^{\prime}} \\
{\left[\left(J_{\mathrm{z}}^{\prime}-J_{\mathrm{x}}^{\prime}\right) \Omega_{\mathrm{z}}^{\prime} \Omega_{\mathrm{x}}^{\prime}+N_{\mathrm{ay}}^{\prime}\right] / J_{\mathrm{y}}^{\prime}} \\
{\left[\left(J_{\mathrm{x}}^{\prime}-J_{\mathrm{y}}^{\prime}\right) \Omega_{\mathrm{x}}^{\prime} \Omega_{\mathrm{y}}^{\prime}+N_{\mathrm{az}}^{\prime}\right] / J_{\mathrm{z}}^{\prime}}
\end{array}\right]
\end{aligned}
$$

By integrating these equations with respect to time, the time course of position of center of mass, flight speed, attitude, and angular velocities are obtained. Fourthorder Runge-Kutta method is used for time integration. The variable $\mathrm{d} t_{\mathrm{dyn}}$ is set to be a fraction $\mathrm{d} t / N$, and the equations of motion are solved $N$ times for each time interval $\mathrm{d} t$ of the flow solver.

\section{Coupling of the two solvers}

Aerodynamic force is obtained by integrating the pressure and shear stress over flier surfaces. Similarly, aerodynamic torque around the center of mass of the flier is obtained. The force and torque are then introduced into the dynamics solver, together with the gravitational contribution to the force. The equations of

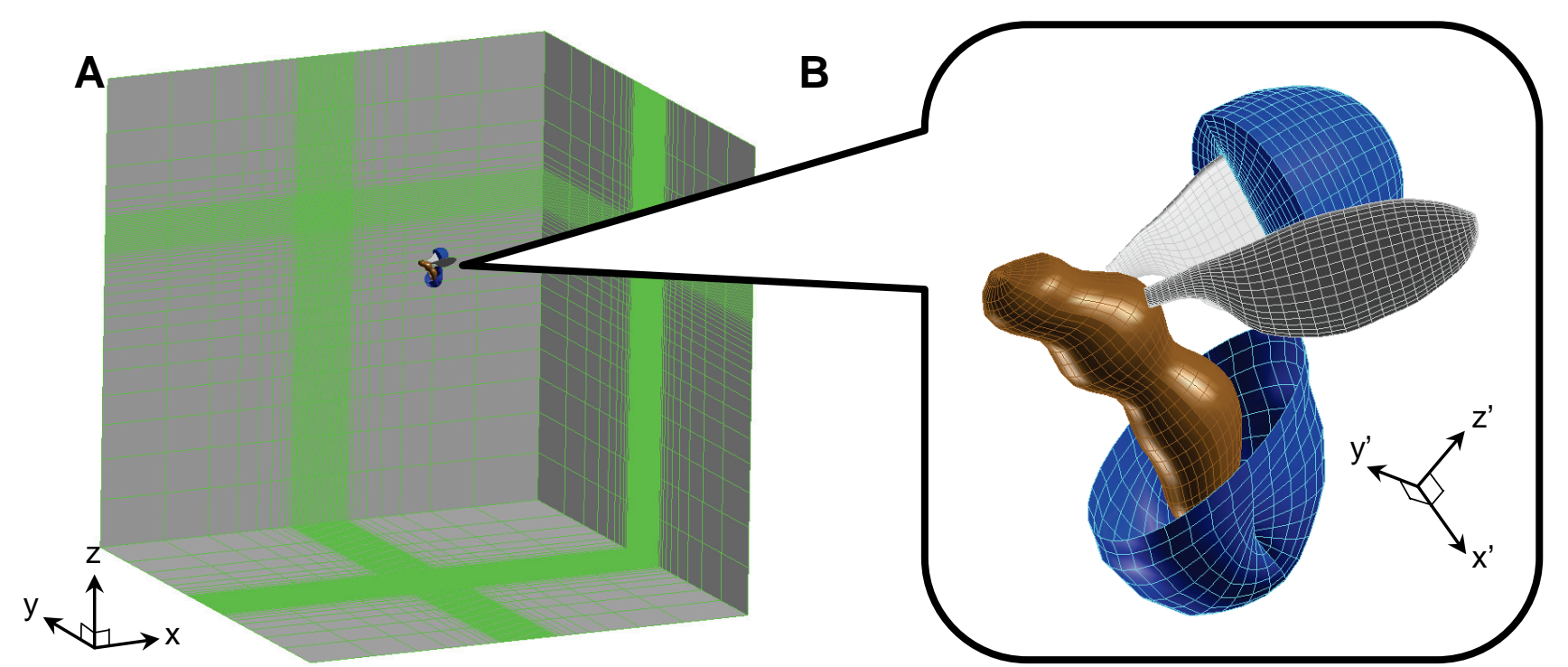

Fig. 2 Grid system. (A) Background grid block. The axis marker indicates global coordinates. (B) Local grid blocks. The axis marker indicates body coordinates. motion (EOM) are solved and the boundary of the flier grid is set to the new position of the flier. The above cycle is iterated. As already mentioned, when solving rigid body dynamics, $\mathrm{d} t_{\mathrm{dyn}}$ is set to be a fraction of $\mathrm{d} t$ for flow field calculation and the EOM are solved several times until the same period as flow solver (i.e. $\mathrm{d} t$ ) passes. For example, if $\mathrm{d} t_{\mathrm{dyn}}=\mathrm{d} t / 10$, then RBD part is computed 10 times with the same aerodynamic forces and torques as inputs. Formerly, the stroke plane angle (SPA) and the body angle (BA) of the flier were set independently. However, in the modified solver presented here, both BA and SPA are determined by the aerodynamic torque. Instead, anatomical stroke plane angle (ASPA), the SPA relative to body, is selected for each wingbeat cycle. See Fig. 3A for the definitions of angles.

For flow field computation, flier grids in global coordinates are necessary. For this purpose, a transformation matrix using unit quaternions is used:

$$
\begin{aligned}
& \underset{\mathrm{b} \rightarrow \mathrm{g}}{\mathbf{C}}=\left[\begin{array}{cc}
\varepsilon_{0}^{2}+\varepsilon_{1}^{2}-\varepsilon_{2}^{2}-\varepsilon_{3}^{2} & 2\left(\varepsilon_{1} \varepsilon_{2}-\varepsilon_{3} \varepsilon_{0}\right) \\
2\left(\varepsilon_{1} \varepsilon_{2}+\varepsilon_{3} \varepsilon_{0}\right) & \varepsilon_{0}^{2}-\varepsilon_{1}^{2}+\varepsilon_{2}^{2}-\varepsilon_{3}^{2} \\
2\left(\varepsilon_{1} \varepsilon_{3}-\varepsilon_{2} \varepsilon_{0}\right) & 2\left(\varepsilon_{2} \varepsilon_{3}+\varepsilon_{1} \varepsilon_{0}\right)
\end{array}\right. \\
& 2\left(\varepsilon_{1} \varepsilon_{3}+\varepsilon_{2} \varepsilon_{0}\right) \\
& 2\left(\varepsilon_{2} \varepsilon_{3}-\varepsilon_{1} \varepsilon_{0}\right) \\
& \varepsilon_{0}^{2}-\varepsilon_{1}^{2}-\varepsilon_{2}^{2}+\varepsilon_{3}^{2}
\end{aligned}
$$

Let the position of a grid point in body and in global coordinates be $\mathbf{R}_{\text {grid }}^{\prime}$ and $\mathbf{R}_{\text {grid }}$, respectively. Therefore,

$$
\mathbf{R}_{\text {grid }}=\underset{\mathrm{b} \rightarrow \mathrm{g}}{\mathbf{C}} \mathbf{R}_{\text {grid }}^{\prime}+\mathbf{R} .
$$




\section{Grid system}

Based on Liu [17], the grid system was modified. Four grid blocks are prepared. Local blocks, which consist of a body grid block and two wing grid blocks, are embedded in a Cartesian background grid block (Fig. 2). The global block is a cube and each side has 60 times the mean chord length. The central region of the global block has uniform spacing and distal region has non-uniform spacing. The flier blocks are placed inside of the uniform mesh region.

The origin in global coordinates locates in the middle of the xy-plane and 40 times the mean chord length above the lower plane. During free-flight computation, if the CM is going to cross the face of the global-grid cell it is in, remeshing occurs so that the flier blocks are always kept inside of the uniform mesh region, thus maintaining the quality of interpolation between local and global blocks.

\section{E. Simplified body oscillation}

Simplified body oscillation due to flapping was implemented. Wing kinematics was set to be symmetric about the x'z'-plane. In addition, rotational motion was neglected for further simplicity. Therefore, the problem is essentially treated as two point masses in the $x$ ' $z$ ' plane. If there is no external force, the translational equation of motion for the flier's center of mass is

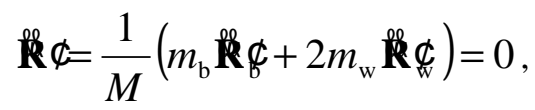

where $m_{\mathrm{b}}$ and $m_{\mathrm{w}}$ are masses of body and wing, respectively. $\mathbf{R}^{\prime}, \mathbf{R}_{\mathrm{b}}^{\prime}$, and $\mathbf{R}_{\mathrm{w}}^{\prime}$ are position vectors (in body coordinates) of the flier's CM, body CM, and wing $\mathrm{CM}$, respectively. Note that we are projecting onto the $x$ 'z'-plane; the resulting vectors are twodimensional and have only $x^{\prime}$ and $z$ ' components. Therefore, the vectors for wings are identical and there is no need to distinguish one from the other. Therefore,

$$
\mathbf{R}_{\mathrm{b}}=-\frac{m_{\mathrm{w}}}{m_{\mathrm{b}}} \mathbf{R}_{\mathrm{w}}^{\mathbb{V}_{\mathrm{w}}} .
$$

By twice integrating both sides with respect to time, body position is obtained as

$$
\mathbf{R}_{\mathrm{b}}^{\prime}=-\frac{m_{\mathrm{w}}}{m_{\mathrm{b}}} \mathbf{R}_{\mathrm{w}}^{\prime} .
$$

\section{F. Wingbeat kinematics manipulation}

In the present study, we attempt to achieve the condition of stable hovering. To realise this condition, some kind of control input is necessary. We chose the following three parameters: wingbeat amplitude $\Phi$, mean of positional angle $\bar{\phi}$, and anatomical stroke plane angle $\theta_{\text {ASPA }}$ (Fig. 3). These values can take certain values for each wingbeat cycle. For transition from one wingbeat to the next, an interpolation method utilizing the hyperbolic tangent function is used, which is employed in the parametric study by Hedrick et al [2].

\section{RESULTS}

A fruit fly (Drosophila melanogaster) model was used for simulation. The body and two wings were assumed to have uniform density. The number of grid points and basic parameters are listed in tables 1 and 2 . Of them, mean chord length, wing length and wingbeat frequency are taken from Hedrick et al. [13].
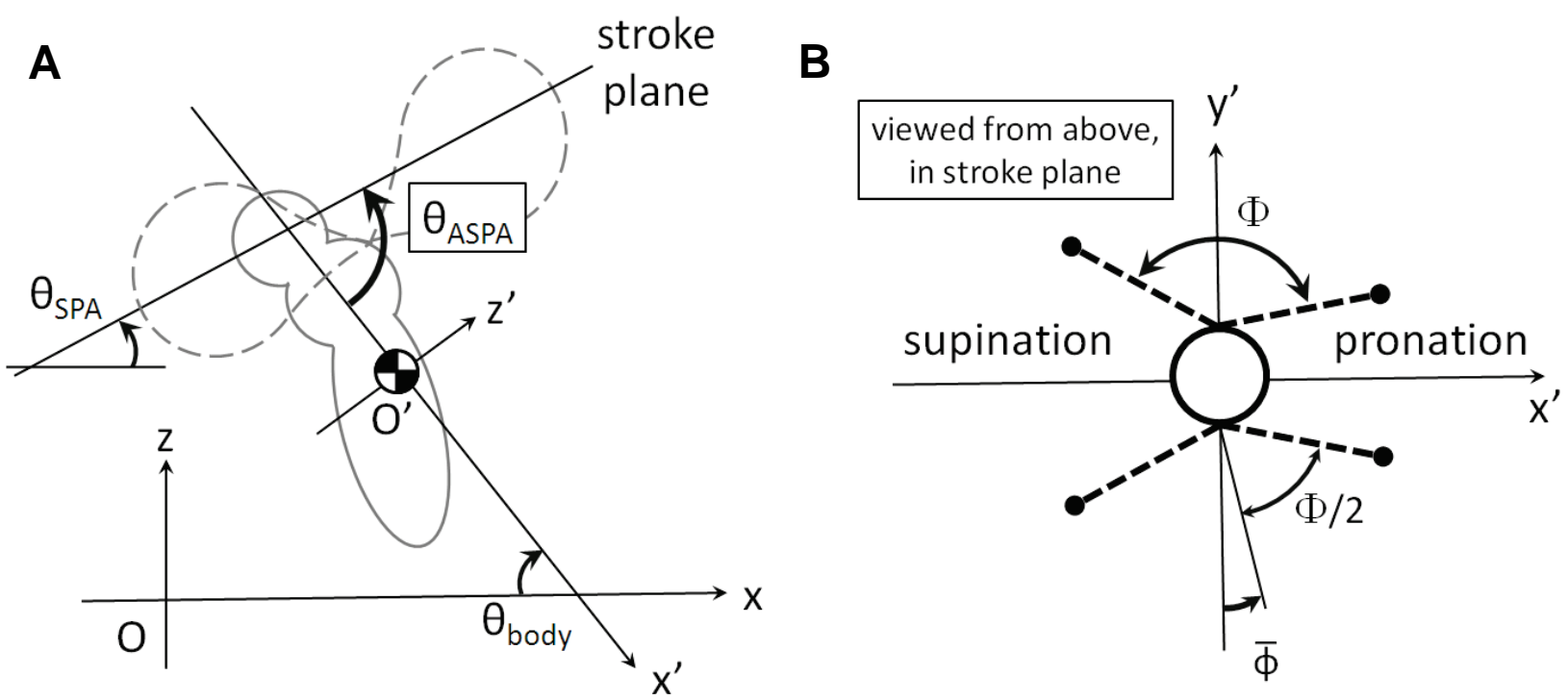

Fig. 3 Kinematic parameters. Left view (A) and top view (B). Three kinematic parameters manipulated in the freeflight Case 2 are shown: anatomical stroke plane angle (ASPA), $\theta_{\text {ASPA }}$, wingbeat amplitude, $\Phi$, and mean stroke plane angle, $\bar{\phi}$. In (A), $\theta_{\mathrm{BA}}$ and $\theta_{\mathrm{BA}}$ are body angle and stroke plane angle, respectively. 
At time $t=0$, flow velocity and pressure are zero everywhere. A no-slip condition is applied to the flier surface and zero-gradient condition is taken for velocity at the background grid block outer boundary.

The body of the insect was fixed in space until the end of the fifth wingbeat cycle, when it was released. In practice, this was done as follows. At first, only the flow field was solved while the body is fixed in space (tethered-flight). At the end of fifth wingbeat cycle ( $t / T$ $=5.0$ ), the flow field data was stored up. The stored data was then used as the initial condition of the new computation, where fluid dynamics and rigid body dynamics are coupled (free-flight). The free-flight computation lasted seven wingbeat cycles.

Here we show the results of two free-flight cases. Both of them used the same stored data of the tetheredflight. In Case 1, the kinematic parameters are unchanged from the tethered-flight computation. In Case 2, on the other hand, the parameters are adjusted manually in some cycles, in which the parameter manipulation was determined in a manner of trial-anderror. The history of kinematic parameters is shown in Table 3. The plots for the kinematics are shown in Figs. 4 and 5.

Since the flight condition was hovering and wing kinematics was symmetric, the motion of flier was confined to the $x z$-plane (as well as the $x^{\prime} z$ '-plane), although the RBD solver itself has 6 degrees of freedom.

The history of forces and velocities at the CM ( $\mathrm{x}-$ and $\mathrm{z}$ - components), and aerodynamic torque and angular velocity (y'-component) around the $\mathrm{CM}$ are shown in Fig. 6. CM position (x- and z-components) and pitch angle are shown in Fig. 7. Fig. 8 shows the body positions and attitudes at $t / T=5$ and $t / T=10$ for both Case 1 and Case 2.

\begin{tabular}{ccc} 
& Table 1 & \multicolumn{2}{c}{ Number of grid points } \\
\hline Body & local grid & $33 \times 33 \times 9$ \\
right wing & local grid & $33 \times 35 \times 13$ \\
left wing & local grid & $33 \times 35 \times 13$ \\
Cartesian & background grid & $61 \times 69 \times 53$ \\
\hline
\end{tabular}

The numbers are for the $\mathrm{i}, \mathrm{j}$, and $\mathrm{k}$ directions, respectively.

\begin{tabular}{|c|c|c|c|}
\hline Table 2 & \multicolumn{2}{|c|}{ Parameters } & \\
\hline Mean chord length & $c_{\mathrm{m}}$ & 0.80 & $\mathrm{~mm}$ \\
\hline Wing length & $R$ & 2.39 & $\mathrm{~mm}$ \\
\hline Wingbeat frequency & $f$ & 218 & $\mathrm{~Hz}$ \\
\hline Mass of flier & $M$ & 0.96 & $\mathrm{mg}$ \\
\hline \multicolumn{4}{|l|}{ Moment of inertia } \\
\hline x'-component & $J_{\mathrm{x}}{ }^{\prime}$ & $7.08 \times 10^{-14}$ & $\mathrm{~kg} \mathrm{~m}^{2}$ \\
\hline y'-component & $J_{\mathrm{y}}{ }^{\prime}$ & $1.12 \times 10^{-12}$ & $\mathrm{~kg} \mathrm{~m}^{2}$ \\
\hline z'-component & $J_{\mathrm{z}}{ }^{\prime}$ & $1.10 \times 10^{-12}$ & $\mathrm{~kg} \mathrm{~m}^{2}$ \\
\hline $\begin{array}{l}\text { Acceleration due to } \\
\text { gravity }\end{array}$ & $g$ & 9.80665 & $\mathrm{~m} / \mathrm{s}^{2}$ \\
\hline Density of air & $\rho$ & 1.225 & $\mathrm{~kg} / \mathrm{m}^{3}$ \\
\hline Kinematic viscosity of air & $v$ & $1.5 \times 10^{-5}$ & $\mathrm{~m}^{2} / \mathrm{s}$ \\
\hline Reference velocity & $u_{\text {ref }}$ & 2.41 & $\mathrm{~m} / \mathrm{s}$ \\
\hline Reynolds number & $\mathrm{Re}$ & 129 & \\
\hline Reduced frequency & $\mathrm{k}$ & 0.23 & \\
\hline
\end{tabular}

Parameters $c_{\mathrm{m}}, R, f$, and $M$ are retrieved from Table 1 of Hedrick et al. [13].

Table 3 History of kinematic parameters

\begin{tabular}{cccc}
\hline cycle & $\Phi$ & $\bar{\phi}$ & $\theta_{\text {ASPA }}$ \\
\hline Tethered: 1-5 & 132.5 & 8.0 & 50 \\
\hline Free-flight Case 1: 6-12 & & & \\
\hline Free-flight Case 2: & 137.5 & 11.7 & 62 \\
6 & 144.0 & 6.5 & 50 \\
7 & 134.3 & 7.6 & 47 \\
8 & 136.5 & 8.0 & 47 \\
$9-12$ & & &
\end{tabular}

All the angles are in degrees.

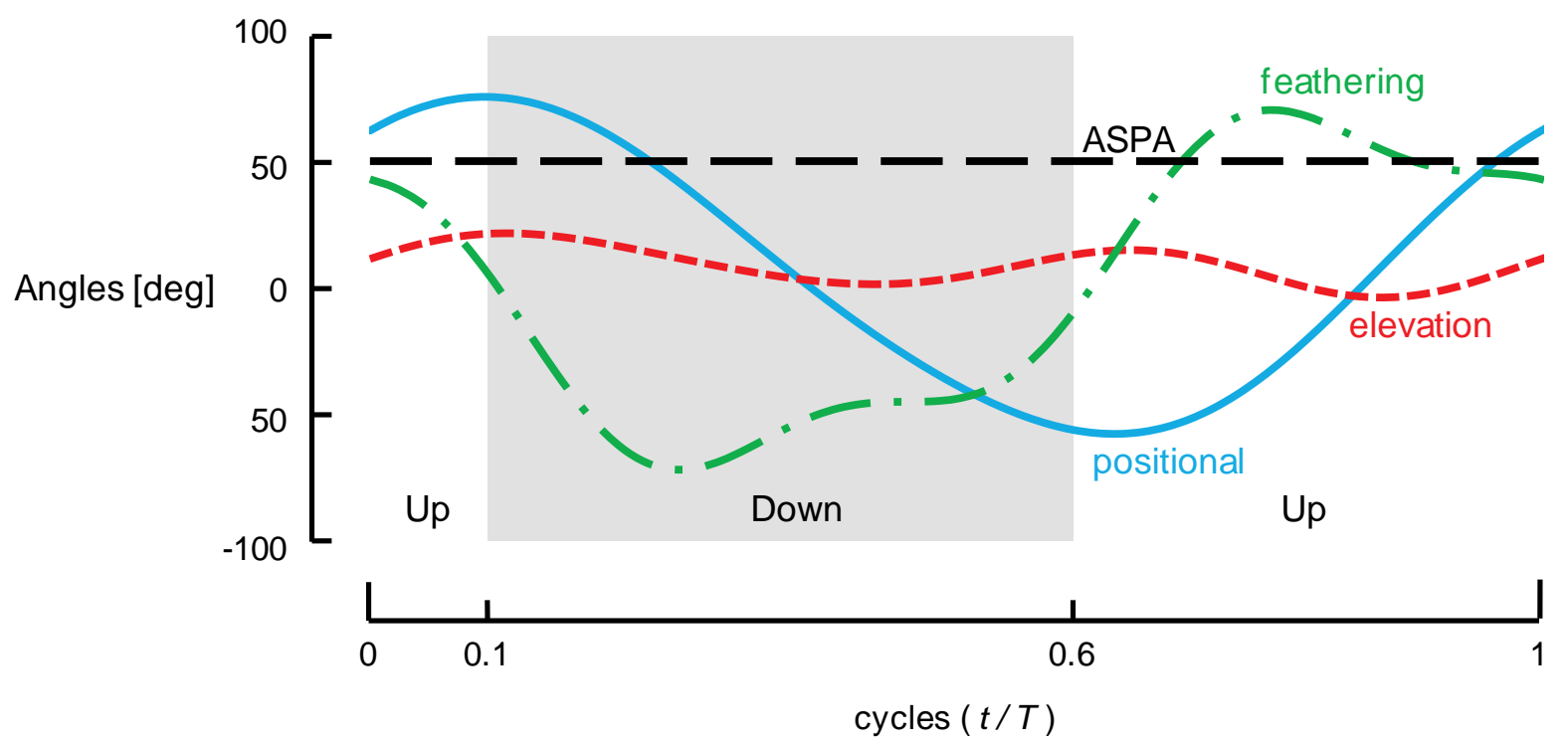

Fig. 4 Basic wing kinematics. The positional angle (blue), the elevation angle (red), the feathering angle (green), and anatomical stroke plane angle (ASPA) (black). Modified from Aono et al. [4]. Although the kinematics for only one cycle is shown, the same kinematics is used during tethered-flight as well as free-flight Case 1 simulation. Note the wingbeat phase is shifted 0.1 cycle so each wingbeat cycle begins with the end of upstroke. This is introduced to keep smoothness in the change of angles in Case 2. 


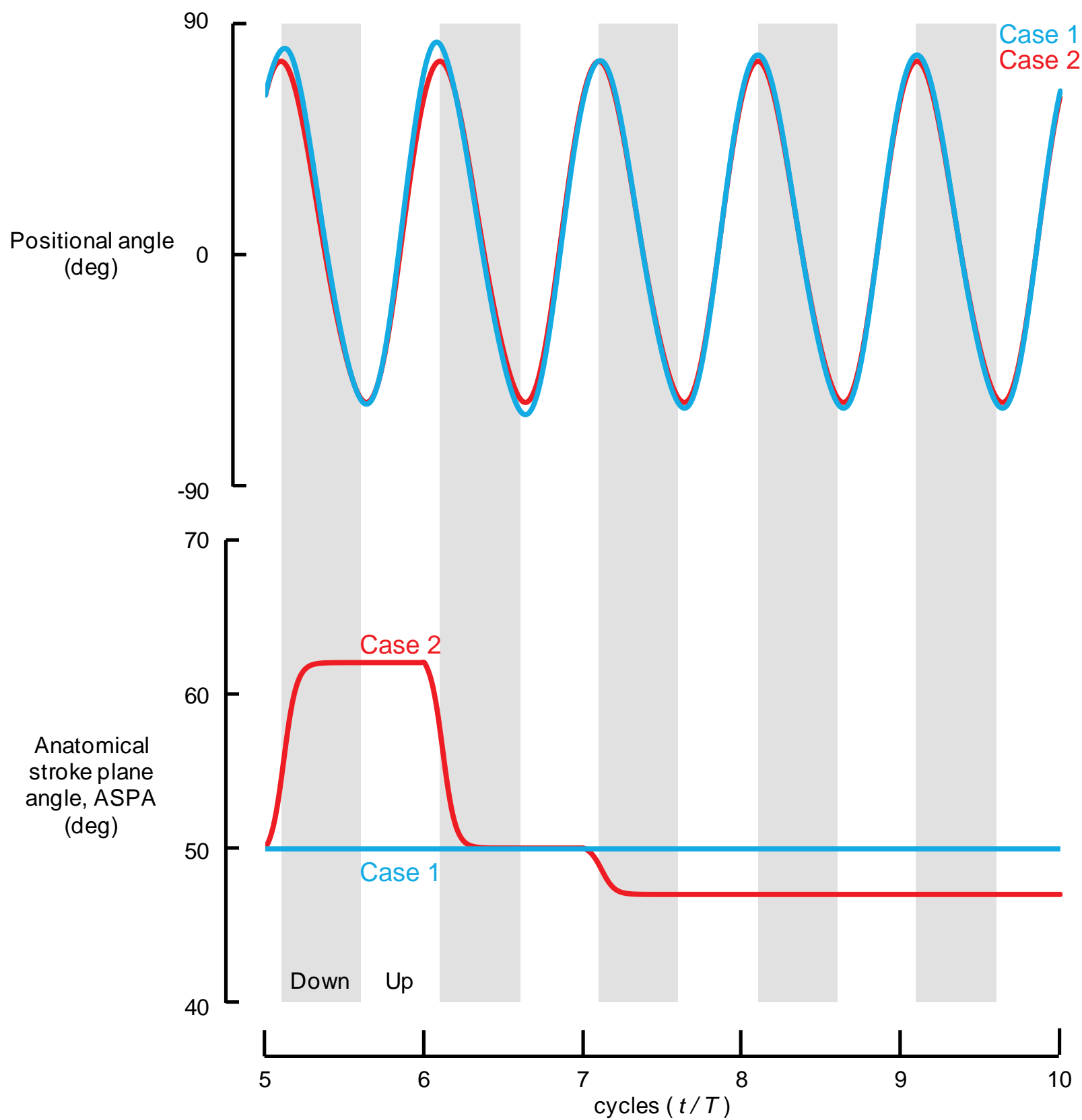

Fig. 5 Prescribed time courses of flapping kinematics in Case 1 (blue) and Case 2 (red). The positional angle and the anatomical stroke plane angle (ASPA). Note both angles are for free-flight.

\section{DISCUSSION}

Although the difference in aerodynamic forces and torques between Case 1 and Case 2 does not seem to be large (Fig. 6), the resultant body trajectories and attitudes differ substantially (Figs. 7 and 8). At $t / T=10$ in Case 1, uncontrolled case, the deviation from the original position is approximately $50 \%$ of the mean chord length with respect to both $\mathrm{x}$ - and z-directions. On the other hand, in Case 2, the manipulated case, overall change in body position was smaller, although pitch angle at first decreased, and then increased back to the original value. This suggests that a cycle-by-cycle manipulation of just three parameters is very likely sufficient to maintain stable hovering for a fruit fly.
This finding is consistent with the study on the freeflight of a fruit fly by Fontaine et al. [19], where it is discussed that the wing kinematics may be adjusted for each wingbeat cycle.

The simulation platform developed in the present study may be a useful tool in investigating the flight performance or obtaining the flight envelope of an insect, or a flapping wing micro air vehicle (MAV).

Future work may include the development of a more realistic RBD solver for larger wing-body mass ratio fliers such as butterflies, or the integration of a fluid structure interaction (FSI) solver to enable passive deformation of the wings. 


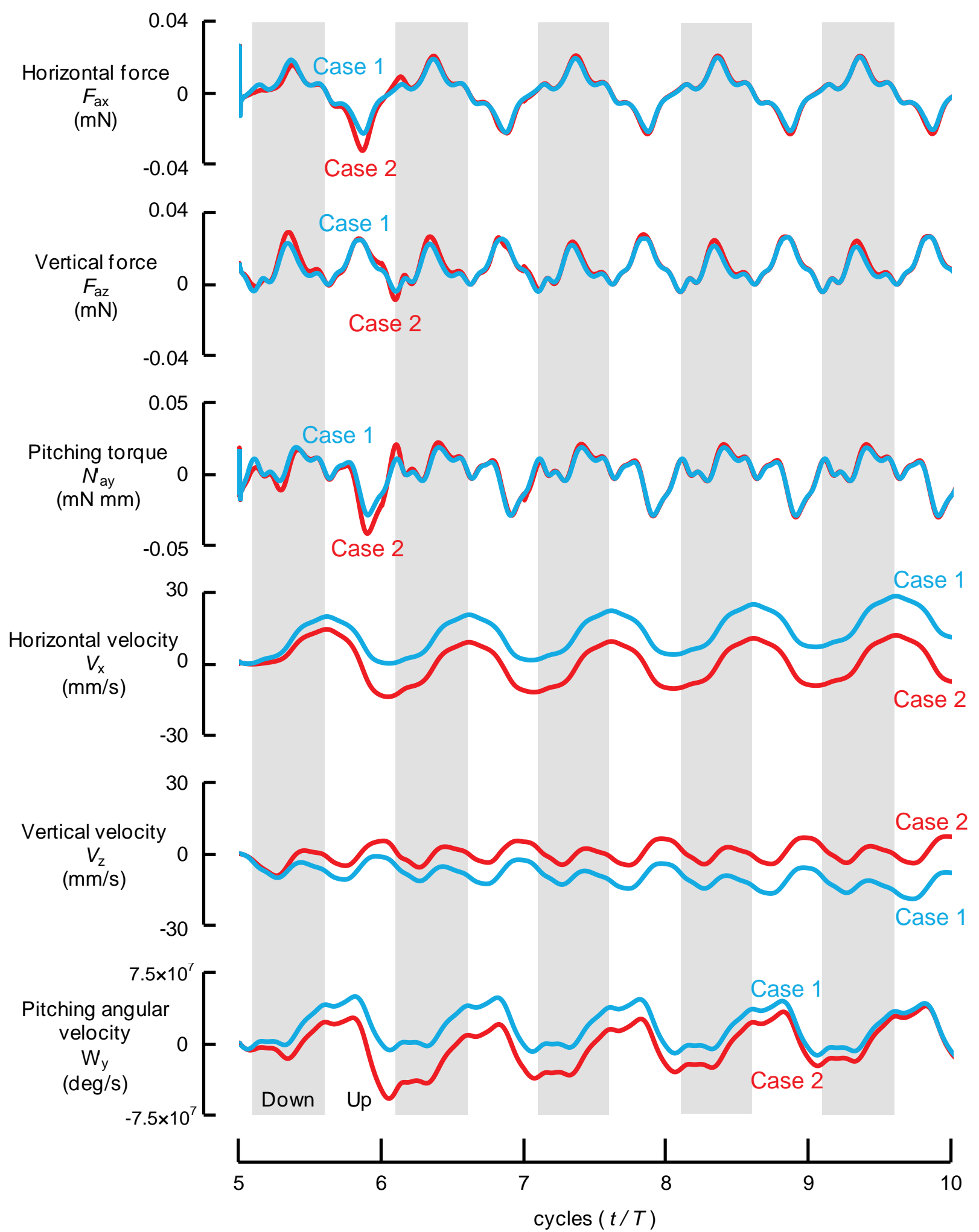

Fig. 6 Time series of flier's performance in Case 1 (blue) and Case 2 (red). The x- and z-components of aerodynamic forces, $F_{\text {ax }}$ and $F_{\text {ax }}$, the y'-component (pitching) of aerodynamic torque around the flier's center of mass in body coordinates, $N_{\text {ay }}$, the x- and zcomponents of velocity at the flier's center of mass in global coordinates, $V_{\mathrm{x}}$ and $V_{\mathrm{z}}$, and the y'-component (pitching) of angular velocity around the center of mass in body coordinates, $\Omega$ ' ${ }_{\mathrm{y}}$. 


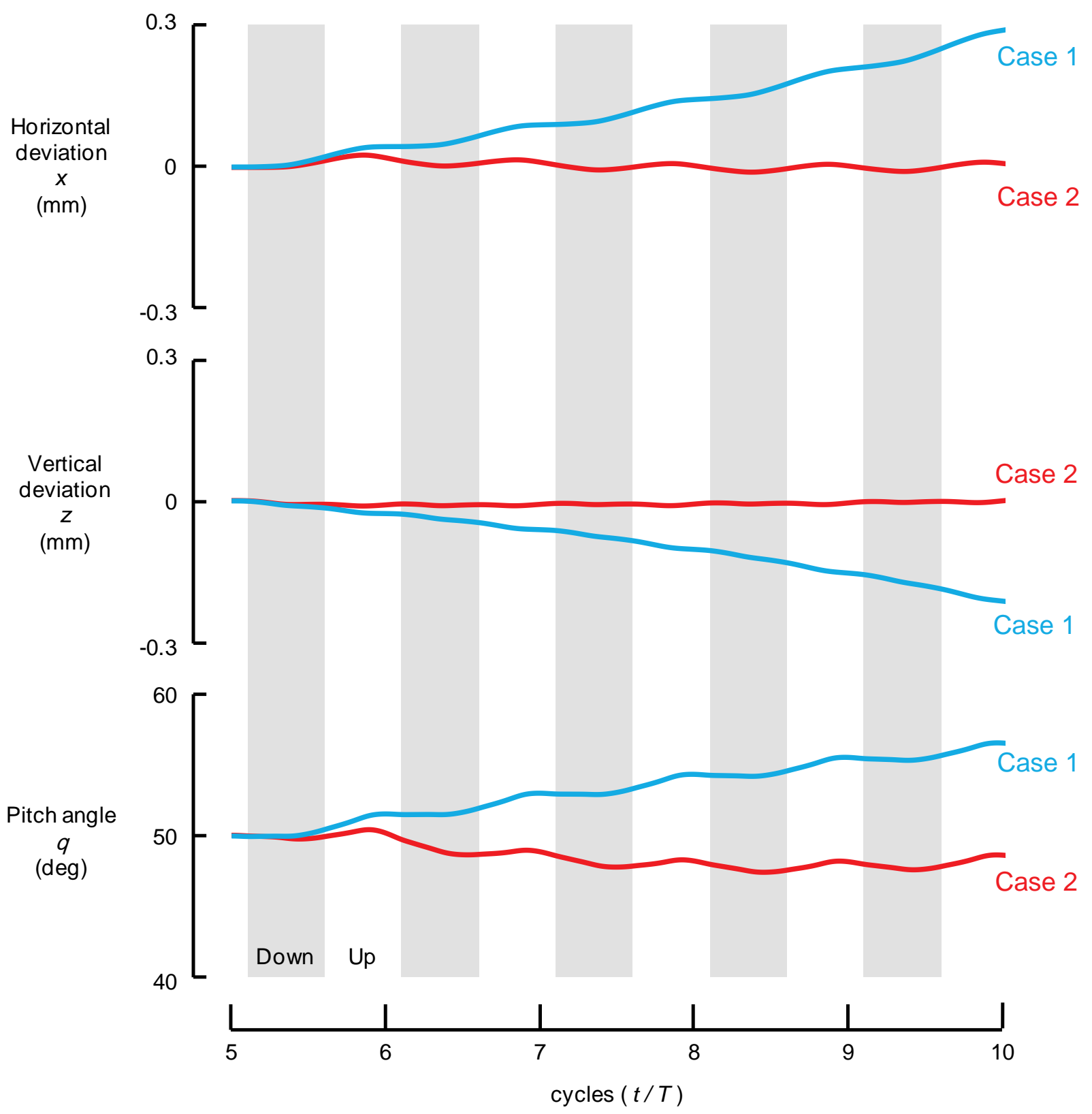

Fig. 7 Time series of flier's motion in Case 1 (blue) and Case 2 (red). The position of center of mass x-component, that of zcomponent, and the pitch angle around the center of mass. Note that the pitch angle is essentially the same as the body angle with current definition.

A

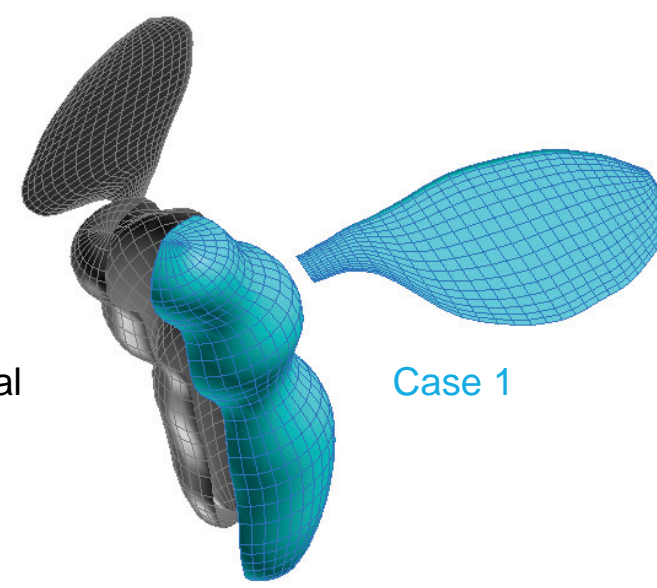

B

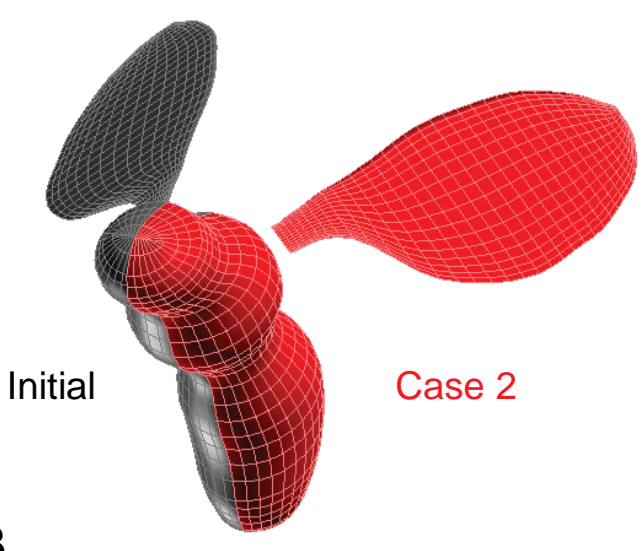

Fig. 8 Body position and attitude. In either (A) and (B), right half of the flier (black) is at $t / T=5.0$, the beginning of free-flight. In (A), left half of the flier (blue) is at $t / T=10.0$ in Case 1. In (B), left half of the flier (red) is at $t / T=10.0$ in Case 2. 


\section{ACKNOWLEDGEMENTS}

This work was partly supported by the Grant-in-Aid for Scientific Research of Nos. 18656056 and 18100002, JSPS, Japan. MM thanks Dr. H. Aono of University of Michigan for the fruit fly data and comments on the manuscript.

\section{REFERENCES}

[1] Sane, S. P. and Dickinson, M. H., The aerodynamic effects of wing rotation and a revised quasi-steady model of flapping flight, Journal of Experimental Biology, Vol. 205 (2002), pp. 1087-1096.

[2] Hedrick, T. L. and Daniel, T. L., Flight control in the hawkmoth Manduca sexta: the inverse problem of hovering, Journal of Experimental Biology, Vol. 209 (2006), pp. 31143130.

[3] Żbikowski, R., Ansari, S. A. and Knowles, K., On mathematical modeling of insect flight dynamics in the context of micro air vehicles, Bioinspiration and Biomimetics, Vol. 1 (2006), pp. R26-R37.

[4] Aono, H., Liang, F. and Liu, H., Near- and far-field aerodynamics in insect hovering flight: an integrated computational study, Journal of Experimental Biology, Vol. 211 (2008), pp. 239-257.

[5] Aono, H., Shyy, W. and Liu, H., Near wake vortex dynamics of a hovering hawkmoth, Acta Mechanica Sinica Vol. 25 (2008), pp. 23-36.

[6] Liu, H. and Aono, H., Size effects on insect hovering aerodynamics: an integrated computational study, Bioinspiration and Biomimetics, Vol. 4 (2009), 015002 (13pp).

[7] Ramamurti, R. and Sandberg, W. C., A computational investigation of the three-dimensional unsteady aerodynamics of Drosophila hovering and maneuvering, Journal of Experimental Biology, Vol. 210 (2007), pp. 881-896.

[8] Sun, M., Wang, J. and Xiong, Y., Dynamic flight stability of hovering insects, Acta Mechanica Sinica, Vol. 23 (2007), pp. 231-246.

[9] Wu, J. H., Zhang, Y. L. and Sun, M., Hovering of model insect: simulation by coupling equations of motion with Navier-Stokes equations, Journal of Experimental Biology, Vol. 212 (2009), pp. 3313-3329.

[10] Zhang, Y. and Sun, M., Dynamic flight stability of a hovering model insect: lateral motion, Acta Mechanica Sinica, DOI: 10.1007/s10409-009-0303-1 (2009).

[11] Card, G. and Dickinson, M., Performance trade-offs in the flight initiation of Drosophila, Journal of Experimental Biology, Vol. 211 (2008), pp. 341-353.
[12] Zabala, F. A., Card, G. M., Fontaine, E. I., Dickinson, M. H. and Murray, R. M., Flight Dynamics and Control of Evasive Maneuvers: The Fruit Fly's Takeoff, IEEE Transactions of Biomedical Engineering, Vol. 56 (2009), pp. 2295-2298.

[13] Hedrick, T. L., Cheng, B. and Deng, X., Wingbeat Time and the Scaling of Passive Rotational Damping in Flapping Flight, Science, Vol. 324 (2009), pp. 252-255.

[14] Ristropha, L., Bergoua, A. J., Ristrophb, G., Coumesc, K., Bermana, G. J., Guckenheimerd, J., Jane Wang, Z. and Cohena, I., Discovering the flight autostabilizer of fruit flies by inducing aerial stumbles, Proceedings of the National Academy of Science, U.S.A., Vol. 107 (2010), pp. 4820-4824.

[15] Kawamura, K., Ueno, Y. and Nakamura, Y., Flight Simulation of Taketombo Based on Computational Fluid Dynamics and Computational Flight Dynamics (in Japanese), Transactions of the Japanese Society of Aeronautical and Space Science, Vol. 56 (2008), pp. 324-330.

[16] Liu, H., Ellington, C. P., Kawachi, K., Van den Berg, C. and Willmott, A. P., A computational fluid dynamic study of hawkmoth hovering, Journal of Experimental Biology, Vol. 201 (1998), pp. 461-477.

[17] Liu, H., Integrated modeling of insect flight: From morphology, kinematics to aerodynamics, Journal of Computational Physics, Vol. 228 (2009), pp. 439-459.

[18] H. Tajima, Fundamentals of Multibody Dynamics (in Japanese), Tokyo, Japan: Tokyo Denki University Press, 2006.

[19] Fontaine, E. I., Zabala, F., Dickinson, M. H. and Burdick, J. W., Wing and body motion during flight initiation in Drosophila revealed by automated visual tracking, Journal of Experimental Biology, Vol. 212 (2009), pp. 1307-1323. 